\title{
Ent-Rosane and Labdane Diterpenoids from Sagittaria sagittifolia with Antibacterial Activity against Three Oral Pathogens
}

Xueting Liu, ${ }^{\dagger}$ Qin Pan, ${ }^{\dagger}$ Yao Shi, ${ }^{\dagger}$ Ian D. Williams,${ }^{\S}$ Herman H-Y Sung, ${ }^{\S}$ Qiong

Zhang, ${ }^{\dagger}$ Jingyu Liang, ${ }^{\dagger}$ Nancy Y. Ip, ${ }^{\perp}$ and Zhida Min*, ${ }^{\dagger}$

Department of Natural Medicinal Chemistry, China Pharmaceutical University, Nanjing 210009, People's Republic of China, $R$ \& D Center, Zhongxin Pharmaceuticals, Tianjin 300458, People's Republic of China, Department of chemistry, The Hong Kong University of Science and Technology, Hong Kong, People's Republic of China, and Department of Biochemistry and Biotechnology Research Institute, The Hong Kong University of Science and Technology, Hong Kong, People's Republic of China 
Table S1. NMR spectral data of compounds $1-3$ in $\mathrm{CDCl}_{3}\left[{ }^{1} \mathrm{H} 400 \mathrm{MHz},{ }^{13} \mathrm{C} 100 \mathrm{MHz}, \mathrm{TMS}, \quad \delta \quad(\mathrm{J}=\mathrm{Hz})\right]$

\begin{tabular}{|c|c|c|c|c|c|c|c|c|c|c|c|c|c|c|c|}
\hline \multirow[t]{2}{*}{ position } & \multicolumn{5}{|c|}{1} & \multicolumn{5}{|c|}{2} & \multicolumn{5}{|c|}{3} \\
\hline & ${ }^{1} \mathrm{H}$ & ${ }^{13} \mathrm{C}$ & HMBC & ${ }^{1} \mathrm{H}-{ }^{1} \mathrm{H} \operatorname{COSY}$ & $\overline{\text { NOESY }}$ & ${ }^{1} \mathrm{H}$ & ${ }^{13} \mathrm{C}$ & HMBC & ${ }^{1} \mathrm{H}-{ }^{1} \mathrm{H}$ COSY & $\overline{\text { NOESY }}$ & ${ }^{1} \mathrm{H}$ & ${ }^{13} \mathrm{C}$ & HMBC & ${ }^{1} \mathrm{H}-{ }^{1} \mathrm{H}$ & $\overline{\text { NOESY }}$ \\
\hline 1a & $1.78 \mathrm{dd}(3.2,12.8)$ & 25.6 & $\mathrm{C}-5,10$ & $\mathrm{H}-1 \mathrm{~b}, 2 \mathrm{a}, 6,10$ & $\mathrm{H}-1 \mathrm{~b}$, & $1.76 \mathrm{~d}(12.4)$ & 25.8 & C-5,9 & $\mathrm{H}-2 \mathrm{a}, 2 \mathrm{~b}, 3 \mathrm{~b}$ & $\mathrm{H}-1 \mathrm{~b}$ & $1.78 \mathrm{~d}(13.2)$ & 25.8 & $\mathrm{C}-5,10$ & $\mathrm{H}-10$ & $\mathrm{H}-1 \mathrm{~b}$ \\
\hline $1 \mathrm{~b}$ & $1.07 \mathrm{~d}(9.6)$ & & $\mathrm{C}-2,10$ & $\mathrm{H}-1 \mathrm{a}, 2 \mathrm{a}, 10$ & $\mathrm{H}-1 \mathrm{a}, 20$ & $1.01 \mathrm{~m}$ & & & & $\mathrm{H}-1 \mathrm{a}, 20$ & $1.06 \mathrm{~m}$ & & $\mathrm{C}-2,10$ & H-3a, 10 & $\mathrm{H}-1 \mathrm{a}, 20$ \\
\hline $2 \mathrm{a}$ & $1.68 \mathrm{~d}(10.4)$ & 22.9 & & $\mathrm{H}-1 \mathrm{a}, 1 \mathrm{~b}, 2 \mathrm{~b}, 3 \mathrm{a}, 3 \mathrm{~b}$ & $\mathrm{H}-2 \mathrm{~b}$ & $1.53 \mathrm{~m}$ & 21.1 & & H-1a, $3 b, 10$ & & $1.57 \mathrm{~m}$ & 21.2 & & H-3a, 18 & $\mathrm{H}-2 \mathrm{~b}$ \\
\hline $2 \mathrm{~b}$ & $1.45 \mathrm{~d}(11.2)$ & & & $\mathrm{H}-2 \mathrm{a}$ & $\mathrm{H}-2 \mathrm{a}, 10$ & $1.39 \mathrm{~m}$ & & & $\mathrm{H}-\mathrm{la}$ & $\mathrm{H}-10,19 \mathrm{a}$ & $1.41 \mathrm{~m}$ & & & & $\mathrm{H}-2 \mathrm{a}, 10$ \\
\hline $3 a$ & $2.18 \mathrm{~d}(13.2)$ & 37.6 & C- $1,4,5$ & $\mathrm{H}-2 \mathrm{a}, 3 \mathrm{~b}$ & $\mathrm{H}-2 \mathrm{a}, 2 \mathrm{~b}, 3 \mathrm{~b}, 18$ & $1.62 \mathrm{~d}(12.5)$ & 35.0 & $\mathrm{C}-1,5$ & & $\mathrm{H}-18$ & $1.61 \mathrm{~d}(11.6)$ & 35.2 & C- $1,4,5$ & H-1b,2a, 17 & $\mathrm{H}-2 \mathrm{a}, 2 \mathrm{~b}, 3 \mathrm{~b}, 18$ \\
\hline $3 \mathrm{~b}$ & $1.18 \mathrm{~d}(13.2)$ & & C-19 & $\mathrm{H}-2 \mathrm{a}, 3 \mathrm{a}$ & $\mathrm{H}-3 \mathrm{a}$ & $1.06 \mathrm{~d}(12.0)$ & & & $\mathrm{H}-1 \mathrm{a}, 2 \mathrm{a}$ & & $1.10 \mathrm{~d}(13.2)$ & & C-19 & & H-3a \\
\hline 4 & & 48.2 & & & & & 39.7 & & & & & 39.8 & & & \\
\hline 5 & & 139.3 & & & & & 141.2 & & & & & 141.1 & & & \\
\hline 6 & $5.70 \mathrm{~d}(5.6)$ & 121.9 & $\mathrm{C}-4,5,7,8,10$ & $\mathrm{H}-1 \mathrm{a}$ & $\mathrm{H}-18$ & $5.52 \mathrm{t}(2.4)$ & 120.7 & $\mathrm{C}-4,7,8,10$ & $\mathrm{H}-10$ & $\mathrm{H}-7 \mathrm{~b}, 18$ & $5.54 \mathrm{t}(2.8)$ & 120.8 & C- $4,5,7,8,10$ & $\mathrm{H}-7 \mathrm{~b}, 10$ & $\mathrm{H}-18$ \\
\hline $7 \mathrm{a}$ & $1.80 \mathrm{~d}(17.6)$ & 30.1 & $C-5,6$ & & & $1.67 \mathrm{~m}$ & 30.2 & & & & $1.71 \mathrm{~m}$ & 30.3 & C-5,6 & & \\
\hline $7 \mathrm{~b}$ & $1.71 \mathrm{~d}(12.0)$ & & C-5,6,14 & & & $1.65 \mathrm{~m}$ & & & & & $1.65 \mathrm{~m}$ & & C-5,6,14 & $\mathrm{H}-6$ & \\
\hline 8 & $1.56 \mathrm{~m}$ & 35.7 & C-14 & & $\mathrm{H}-10,17$ & $1.42 \mathrm{~m}$ & 35.9 & & H-11a & $\mathrm{H}-17$ & $1.43 \mathrm{~m}$ & 36.0 & C-14 & $\mathrm{H}-12 \mathrm{~b}$ & $\mathrm{H}-10,17$ \\
\hline 9 & & 34.9 & & & & & 34.7 & & & & & 34.8 & & & \\
\hline 10 & $1.95 \mathrm{~d}(12.8)$ & 48.8 & $\mathrm{C}-1$ & $\mathrm{H}-1 \mathrm{a}, 1 \mathrm{~b}$ & $\mathrm{H}-2 \mathrm{~b}, 8,11 \mathrm{~b}$ & $1.83 \mathrm{~d}(12.8)$ & 47.6 & & $\mathrm{H}-2 \mathrm{a}$ & $\mathrm{H}-2 \mathrm{~b}, 11 \mathrm{~b}, 19 \mathrm{a}, 19 \mathrm{~b}$ & $1.86 \mathrm{~d}(11.8)$ & 47.7 & C-1 & $\mathrm{H}-1 \mathrm{a}, 6$ & $\mathrm{H}-2 \mathrm{~b}, 8,11 \mathrm{~b}$ \\
\hline 11a & $1.62 \mathrm{~d}(10.8)$ & 34.0 & C- $-12,20$ & & & $1.61 \mathrm{~d}(13.0)$ & 34.0 & $\mathrm{C}-13$ & $\mathrm{H}-8,12 \mathrm{~b}$ & $\mathrm{H}-1 \mathrm{a}$ & $1.63 \mathrm{~d}(13.2)$ & 34.1 & C- $-12,20$ & & \\
\hline $11 \mathrm{~b}$ & $1.29 \mathrm{~d}(9.6)$ & & $\mathrm{C}-8,12$ & & H-10,17 & $1.20 \mathrm{~d}(12.6)$ & & C-10,14,15 & & $\mathrm{H}-8,17$ & $1.23 \mathrm{~d}(12.0)$ & & C-8,12 & & H-10,17 \\
\hline 12a & $1.47 \mathrm{t}(12.8)$ & 32.3 & C-15,17 & & & $1.43 \mathrm{t}(16.0)$ & 32.3 & C-9 & $\mathrm{H}-12 \mathrm{~b}$ & & $1.45 \mathrm{t}(18.0)$ & 32.3 & C-15,17 & & \\
\hline $12 \mathrm{~b}$ & $1.22 \mathrm{~d}(13.6)$ & & C-14 & & & $1.21 \mathrm{~d}(11.2)$ & & C-14 & $\mathrm{H}-11 \mathrm{a}, 12 \mathrm{a}$ & $\mathrm{H}-15,16 \mathrm{a}, 20$ & $1.22 \mathrm{~d}(10.4)$ & & C-14 & H-8 & \\
\hline 13 & & 36.3 & & & & & 36.1 & & & & & 36.2 & & & \\
\hline $14 \mathrm{a}$ & $1.26 \mathrm{dd}(6.4,12.0)$ & 38.9 & $\mathrm{C}-17$ & & $\mathrm{H}-15,16 \mathrm{a}$ & $1.22 \mathrm{dd}(6.0,14.0)$ & 38.9 & $\mathrm{C}-12,15$ & $\mathrm{H}-14 \mathrm{~b}$ & H-15,16a, 17 & $1.24 \mathrm{dd}(6.0,13.2)$ & 39.0 & $\mathrm{C}-17$ & $\mathrm{H}-14 \mathrm{~b}$ & $\mathrm{H}-15,16 \mathrm{a}$ \\
\hline $14 \mathrm{~b}$ & $1.12 \mathrm{~d}(10.0)$ & & & & & $1.08 \mathrm{~d}(13.2)$ & & & $\mathrm{H}-14 \mathrm{a}$ & $\mathrm{H}-20$ & $1.09 \mathrm{~d}(13.6)$ & & & H-14a & \\
\hline 15 & $5.80 \mathrm{~d}(10.8,17.6)$ & 151.3 & $\mathrm{C}-12,13,14,17$ & $\mathrm{H}-16 \mathrm{a}, 16 \mathrm{~b}$ & H-14a,16a,16b,17 & $5.77 \mathrm{dd}(10.8,17.6)$ & 151.2 & C-12,13,14,17 & H-16a,16b & H-12b,16a,16b,17 & $5.79 \mathrm{dd}(10.8,17.6)$ & 151.3 & C-12,13,14,17 & H-16a,16b & H-14a, 16a, 16b, 17 \\
\hline 16a & $4.90 \mathrm{~d}(17.6)$ & 108.6 & C-13,15 & H-15 & H-14a, 15,17 & $4.87 \mathrm{dd}(1.2,17.2)$ & 108.6 & C-13,15 & $\mathrm{H}-15$ & H-12b,14a, 15,17 & $4.89 \mathrm{dd}(1.2,17.6)$ & 108.6 & C-13,15 & $\mathrm{H}-15$ & H-14a, 15,17 \\
\hline $16 \mathrm{~b}$ & $4.83 \mathrm{~d}(10.8)$ & & C-15 & H-15 & $\mathrm{H}-15$ & $4.80 \mathrm{dd}(1.2,10.8)$ & & C-13,15 & $\mathrm{H}-15$ & $\mathrm{H}-15$ & $4.82 \mathrm{dd}(1.2,10.8)$ & & C-15 & $\mathrm{H}-15$ & $\mathrm{H}-15$ \\
\hline 17 & $1.01 \mathrm{~s}$ & 22.4 & C-12,14,15 & & $\mathrm{H}-8,11 \mathrm{~b}$ & $0.96 \mathrm{~s}$ & 22.2 & C-12,13,14,15 & & $\mathrm{H}-8,14 \mathrm{a}, 15,16 \mathrm{a}$ & $0.98 \mathrm{~s}$ & 22.3 & C-12,14,15 & & $\mathrm{H}-8,11 \mathrm{~b}$ \\
\hline 18 & $1.36 \mathrm{~s}$ & 25.2 & C- $-3,4,5,19$ & & $\mathrm{H}-3 \mathrm{a}, 6$ & $1.02 \mathrm{~s}$ & 24.9 & C-3,5,19 & & $\mathrm{H}-3 \mathrm{a}, 6,19 \mathrm{~b}$ & $1.04 \mathrm{~s}$ & 24.9 & C-3,4,5,19 & $\mathrm{H}-2 \mathrm{a}, 3 \mathrm{a}$ & $\mathrm{H}-3 \mathrm{a}, 6$ \\
\hline 19a & & 183.6 & & & & $3.74 \mathrm{~d}(9.6)$ & 73.7 & $\mathrm{C}-4,5,18,1^{\prime}$ & $\mathrm{H}-19 \mathrm{~b}$ & $\mathrm{H}-10,2 \mathrm{~b}, 19 \mathrm{~b}, 1^{\prime}, 4^{\prime}$ & $3.75 \mathrm{~d}(9.2)$ & 74.0 & C- $4,5,18,1^{\prime}$ & $\mathrm{H}-19 \mathrm{~b}$ & \\
\hline $19 \mathrm{~b}$ & & & & & & $2.99 \mathrm{~d}(9.2)$ & & C-4,5,18,1' & $\mathrm{H}-19 \mathrm{a}$ & $\mathrm{H}-10,18,19 \mathrm{a}, 1^{\prime}$ & $3.07 \mathrm{~d}(9.2)$ & & C- $4,5,18,1^{\prime}$ & $\mathrm{H}-19 \mathrm{a}$ & \\
\hline 20 & $0.67 \mathrm{~s}$ & 12.6 & C- $8,9,10$ & & $\mathrm{H}-1 \mathrm{~b}, 11 \mathrm{a}$ & $0.61 \mathrm{~s}$ & 12.1 & C- 8,10 & & $\mathrm{H}-7 \mathrm{~b}, 12 \mathrm{~b}$ & $0.64 \mathrm{~s}$ & 12.1 & C- $8,9,10$ & & $\mathrm{H}-1 \mathrm{~b}, 11 \mathrm{a}$ \\
\hline $1^{\prime}$ & & & & & & $4.90 \mathrm{br} \mathrm{s}$ & 108.0 & C-19,3' & & H-19a, 19b, $3^{\prime}$ & $4.94 \mathrm{br} \mathrm{s}$ & 108.0 & C-19,3' & & $\mathrm{H}-19 \mathrm{a}, 19 \mathrm{~b}$ \\
\hline $2^{\prime}$ & & & & & & $3.98 \mathrm{~s}$ & 79.2 & $\mathrm{C}-3^{\prime}, 4^{\prime}$ & & $H-19 a, 4^{\prime}$ & $4.06 \mathrm{~d}(1.2)$ & 79.5 & $\mathrm{C}-3^{\prime}, 4^{\prime}$ & $\mathrm{H}-3^{\prime}, 5^{\prime} \mathrm{a}$ & $\mathrm{H}-3 \mathrm{a}, 19 \mathrm{a}$ \\
\hline
\end{tabular}




\begin{tabular}{|c|c|c|c|c|c|c|c|c|c|c|}
\hline $3^{\prime}$ & $3.95 \mathrm{~s}$ & 77.5 & $\mathrm{C}-2^{\prime}$ & $\mathrm{H}-4^{\prime}$ & $\mathrm{H}-5^{\prime \prime} \mathrm{b}$ & $3.86 \mathrm{br} \mathrm{s}$ & 78.0 & $\mathrm{C}-2^{\prime}$ & $\mathrm{H}-2^{\prime}, 4^{\prime}, 5^{\prime} \mathrm{a}$ & $\mathrm{H}-5^{\prime} \mathrm{a}, 5^{\prime} \mathrm{b}$ \\
\hline $4^{\prime}$ & $4.06 \mathrm{~d}(2.8)$ & 86.3 & $\mathrm{C}-3^{\prime}$ & $\mathrm{H}-19 \mathrm{a}, 3^{\prime}, 5^{\prime} \mathrm{b}$ & $\mathrm{H}-19 \mathrm{a}, 5^{\prime} \mathrm{b}$ & $4.19 \mathrm{dd}(2.4,4.0)$ & 84.2 & $\mathrm{C}-3^{\prime}$ & $\mathrm{H}-3^{\prime}$ & $\mathrm{H}-19 \mathrm{~b}$ \\
\hline $5^{\prime} \mathrm{a}$ & $3.82 \mathrm{~d}(2.8)$ & 61.4 & $\mathrm{C}-3^{\prime}, 4^{\prime}$ & & & $4.27 \mathrm{~d}(4.4)$ & 64.1 & $\mathrm{C}-3^{\prime}, 4^{\prime}, 5^{\prime}-\mathrm{CO}$ & $\mathrm{H}-3^{\prime}, 4^{\prime}$ & $\mathrm{H}-3^{\prime}$ \\
\hline $5^{\prime} \mathrm{b}$ & $3.79 \mathrm{~d}(2.4)$ & & $\mathrm{C}-3^{\prime}, 4^{\prime}$ & $\mathrm{H}-4^{\prime}$ & $\mathrm{H}-4^{\prime}$ & $4.27 \mathrm{~d}(4.4)$ & & $\mathrm{C}-3^{\prime}, 4^{\prime}, 5^{\prime}-\mathrm{CO}$ & $\mathrm{H}-3^{\prime}, 5^{\prime} \mathrm{a}$ & H-3a, 19a, $3^{\prime}$ \\
\hline \multicolumn{11}{|l|}{$5^{\prime}-\mathrm{OAc}$} \\
\hline $\mathrm{COCH}_{3}$ & & & & & & & 170.4 & & & \\
\hline $\mathrm{COCH}_{3}$ & & & & & & $2.08 \mathrm{~s}$ & 20.8 & $5^{\prime}-\mathrm{CO}$ & & \\
\hline
\end{tabular}

Table S2. NMR spectral data of compounds 4-6 in $\mathrm{CDCl}_{3}\left[{ }^{1} \mathrm{H} 400 \mathrm{MHz},{ }^{13} \mathrm{C} 100 \mathrm{MHz}, \mathrm{TMS}, \quad \delta \quad(J=\mathrm{Hz})\right]$

\begin{tabular}{|c|c|c|c|c|c|c|c|c|c|c|c|c|c|c|c|}
\hline \multirow[t]{2}{*}{ position } & \multicolumn{5}{|c|}{4} & \multicolumn{5}{|c|}{5} & \multicolumn{4}{|c|}{6} & \multirow[b]{2}{*}{ NOESY } \\
\hline & ${ }^{1} \mathrm{H}$ & ${ }^{13} \mathrm{C}$ & HMBC & ${ }^{1} \mathrm{H}-{ }^{1} \mathrm{H}$ & $\overline{\text { NOESY }}$ & ${ }^{1} \mathrm{H}$ & ${ }^{13} \mathrm{C}$ & HMBC & ${ }^{1} \mathrm{H}-{ }^{1} \mathrm{H} \operatorname{COSY}$ & $\overline{\text { NOESY }}$ & ${ }^{1} \mathrm{H}$ & ${ }^{13} \mathrm{C}$ & HMBC & ${ }^{1} \mathrm{H}-{ }^{1} \mathrm{H} \operatorname{COSY}$ & \\
\hline 1a & $1.77 \mathrm{~d}(12.9)$ & 25.9 & $\mathrm{C}-10$ & $\mathrm{H}-2 \mathrm{a}, 2 \mathrm{~b}, 3 \mathrm{~b}$ & $\mathrm{H}-1 \mathrm{~b}$, & $1.79 \mathrm{~d}(13.0)$ & 25.9 & C-5,10 & $\mathrm{H}-10$ & $\mathrm{H}-1 \mathrm{~b}$ & $1.75 \mathrm{~d}(13.2)$ & 26.0 & C-10 & $\mathrm{H}-10$ & \\
\hline $1 \mathrm{~b}$ & $1.02 \mathrm{~m}$ & & & & $\mathrm{H}-1 \mathrm{a}, 20$ & $1.03 \mathrm{~m}$ & & $\mathrm{C}-2,10$ & $\mathrm{H}-3 \mathrm{a}, 10$ & $\mathrm{H}-1 \mathrm{a}, 20$ & $1.03 \mathrm{~m}$ & & & $\mathrm{H}-3 \mathrm{a}, 10$ & \\
\hline $2 \mathrm{a}$ & $1.55 \mathrm{~m}$ & 21.2 & & $\mathrm{H}-1 \mathrm{a}, 3 \mathrm{~b}, 10$ & $\mathrm{H}-2 \mathrm{~b}$ & $1.55 \mathrm{~m}$ & 21.2 & & $\mathrm{H}-3 \mathrm{a}, 18$ & & $1.51 \mathrm{~m}$ & 21.3 & & $\mathrm{H}-3 \mathrm{a}, 18$ & $\mathrm{H}-3 \mathrm{a}$, \\
\hline $2 \mathrm{~b}$ & $1.41 \mathrm{~m}$ & & $\mathrm{C}-10$ & $\mathrm{H}-1 \mathrm{a}$ & $\mathrm{H}-2 \mathrm{a}, 10$ & $1.44 \mathrm{~m}$ & & & & $\mathrm{H}-10,19 \mathrm{a}$ & $1.44 \mathrm{~m}$ & & C-10 & & $\mathrm{H}-10,19 \mathrm{a}, 19 \mathrm{~b}$ \\
\hline 3a & $1.70 \mathrm{~d}(12.0)$ & 35.0 & $\mathrm{C}-4$ & & $\mathrm{H}-2 \mathrm{a}, 2 \mathrm{~b}, 3 \mathrm{~b}, 18$ & $1.69 \mathrm{~d}(11.5)$ & 35.0 & $\mathrm{C}-1,4,5$ & $\mathrm{H}-1 \mathrm{~b}, 2 \mathrm{a}, 17$ & H-18 & $1.77 \mathrm{~d}(13.2)$ & 34.8 & C-4 & $\mathrm{H}-1 \mathrm{~b}, 2 \mathrm{a}, 17$ & $\mathrm{H}-2 \mathrm{a}, 3 \mathrm{~b}, 18,2^{\prime}, 5^{\prime} \mathrm{b}$ \\
\hline $3 b$ & $1.07 \mathrm{~m}$ & & $\mathrm{C}-2,18$ & $\mathrm{H}-1 \mathrm{a}, 2 \mathrm{a}$ & $\mathrm{H}-3 \mathrm{a}$ & $1.10 \mathrm{~d}(11.9)$ & & C-19 & & & $1.01 \mathrm{~m}$ & & $\mathrm{C}-2,18$ & & H-3a \\
\hline 4 & & 39.9 & & & & & 39.8 & & & & & 39.9 & & & \\
\hline 5 & & 141.2 & & & & & 141.1 & & & & & 141.4 & & & \\
\hline 6 & $5.54 \mathrm{t}(2.4)$ & 120.7 & $\mathrm{C}-4,7,10$ & H-10 & $\mathrm{H}-18$ & $5.54 \mathrm{t}(2.4)$ & 120.8 & C- $4,5,7,8,10$ & $\mathrm{H}-7 \mathrm{~b}, 10$ & $\mathrm{H}-7 \mathrm{~b}, 18$ & $5.54 \mathrm{t}(2.4)$ & 120.5 & $\mathrm{C}-4,7,10$ & $\mathrm{H}-7 \mathrm{~b}, 10$ & $\mathrm{H}-18,19 \mathrm{~b}$ \\
\hline $7 \mathrm{a}$ & $1.68 \mathrm{~m}$ & 30.3 & C-5,6 & & & $1.68 \mathrm{~m}$ & 30.2 & C-5,6 & & & $1.69 \mathrm{~m}$ & 30.3 & C-5,6 & & \\
\hline $7 \mathrm{~b}$ & $1.66 \mathrm{~m}$ & & & & & $1.66 \mathrm{~m}$ & & $C-5,6,14$ & H-6 & & $1.64 \mathrm{~m}$ & & & H-6 & $\mathrm{H}-20$ \\
\hline 8 & $1.43 \mathrm{~m}$ & 36.0 & C-14 & $\mathrm{H}-11 \mathrm{a}$ & $\mathrm{H}-10,17$ & $1.42 \mathrm{~s}$ & 36.0 & C-14 & $\mathrm{H}-12 \mathrm{~b}$ & $\mathrm{H}-17$ & $1.42 \mathrm{~m}$ & 36.0 & $\mathrm{C}-14$ & $\mathrm{H}-12 \mathrm{~b}$ & \\
\hline 9 & & 34.8 & & & & & 34.8 & & & & & 34.7 & & & \\
\hline 10 & $1.84 \mathrm{~d}(12.5)$ & 47.7 & & $\mathrm{H}-2 \mathrm{a}$ & $\mathrm{H}-2 \mathrm{~b}, 8,11 \mathrm{~b}$ & $1.83 \mathrm{~d}(12.0)$ & 47.7 & C-1 & $\mathrm{H}-1 \mathrm{a}, 6$ & $\mathrm{H}-2 \mathrm{~b}, 11 \mathrm{~b}, 19 \mathrm{a}, 19 \mathrm{~b}$ & $1.86 \mathrm{~d}(12.8)$ & 47.8 & & $\mathrm{H}-1 \mathrm{a}, 6$ & \\
\hline $11 \mathrm{a}$ & $1.63 \mathrm{~d}(12.6)$ & 34.0 & C- $10,13,20$ & $\mathrm{H}-8,12 \mathrm{~b}$ & & $1.64 \mathrm{~d}(12.8)$ & 34.0 & $\mathrm{C}-12,20$ & & $\mathrm{H}-1 \mathrm{a}$ & $1.61 \mathrm{~d}(11.7)$ & 34.1 & C-10,13,20 & & \\
\hline $11 \mathrm{~b}$ & $1.23 \mathrm{~d}(13.0)$ & & $\mathrm{C}-20$ & & $\mathrm{H}-10,17$ & $1.23 \mathrm{~d}(11.5)$ & & C-8,12 & & $\mathrm{H}-8,17$ & $1.23 \mathrm{~d}(12.0)$ & & $\mathrm{C}-20$ & & \\
\hline $12 \mathrm{a}$ & $1.45 \mathrm{t}(15.6)$ & 32.3 & C-11,13,17 & $\mathrm{H}-12 \mathrm{~b}$ & & $1.45 \mathrm{t}(15.6)$ & 32.3 & $\mathrm{C}-15,17$ & & & $1.44 \mathrm{t}(13.9)$ & 32.4 & C-11,13,17 & & $\mathrm{H}-12 \mathrm{~b}, 15,16 \mathrm{a}, 17$ \\
\hline $12 \mathrm{~b}$ & $1.23 \mathrm{~d}(11.0)$ & & & $\mathrm{H}-11 \mathrm{a}, 12 \mathrm{a}$ & & $1.21 \mathrm{~d}(11.5)$ & & C-14 & $\mathrm{H}-8$ & $\mathrm{H}-15,16 \mathrm{a}, 20$ & $1.21 \mathrm{~d}(12.2)$ & & & $\mathrm{H}-8$ & $\mathrm{H}-12 \mathrm{a}$ \\
\hline 13 & & 36.2 & & & & & 36.2 & & & & & 36.2 & & & \\
\hline $14 \mathrm{a}$ & $1.23 \mathrm{dd}(5.6,13.2)$ & 39.0 & C-7,13,15,17 & $\mathrm{H}-14 \mathrm{~b}$ & $\mathrm{H}-15,16 \mathrm{a}$ & $1.24 \mathrm{dd}(6.0,13.8)$ & 39.0 & C-17 & $\mathrm{H}-14 \mathrm{~b}$ & H-15,16a, 17 & $1.23 \mathrm{~m}$ & 39.0 & C- $7,13,15,17$ & $\mathrm{H}-14 \mathrm{~b}$ & $\mathrm{H}-15,16 \mathrm{a}$ \\
\hline $14 \mathrm{~b}$ & $1.08 \mathrm{dd}(4.0,13.6)$ & & $\mathrm{C}-13$ & $\mathrm{H}-14 \mathrm{a}$ & & $1.07 \mathrm{~d}(12.9)$ & & & $\mathrm{H}-14 \mathrm{a}$ & $\mathrm{H}-20$ & $1.07 \mathrm{t}(3.2)$ & & $\mathrm{C}-13$ & $\mathrm{H}-14 \mathrm{a}$ & $\mathrm{H}-14 \mathrm{a}, 15,16 \mathrm{a}$ \\
\hline 15 & $5.78 \mathrm{dd}(10.4,17.2)$ & 151.3 & C-12,13,14,17 & H-16a,16b & H-14a, 16a,16b,17 & $5.78 \mathrm{dd}(10.8,17.6)$ & 151.3 & C-12,13,14,17 & $\mathrm{H}-16 \mathrm{a}, 16 \mathrm{~b}$ & $\mathrm{H}-12 \mathrm{~b}, 16 \mathrm{a}, 16 \mathrm{~b}, 17$ & $5.78 \mathrm{dd}(10.8,17.6)$ & 151.3 & C-12,13,14,17 & H-16a, 16b & H-12a, $14 a, 14 b, 16 a, 16 b, 17$ \\
\hline $16 \mathrm{a}$ & $4.89 \mathrm{dd}(1.2,17.6)$ & 108.6 & C-13,15 & H-15 & H-14a, 15,17 & $4.89 \mathrm{dd}(1.2,17.6)$ & 108.6 & C- 13,15 & H-15 & $\mathrm{H}-12 \mathrm{~b}, 14 \mathrm{a}, 15,17$ & $4.89 \mathrm{dd}(1.2,17.6)$ & 108.6 & C- 13,15 & $\mathrm{H}-15$ & H-12a,14a, 14b,15,17 \\
\hline $16 \mathrm{~b}$ & $4.82 \mathrm{dd}(1.2,10.8)$ & & C- $-13,15$ & $\mathrm{H}-15$ & $\mathrm{H}-15$ & $4.82 \mathrm{dd}(1.2,10.2)$ & & C-15 & $\mathrm{H}-15$ & H-15 & $4.81 \mathrm{dd}(1.2,10.4)$ & & C- 13,15 & $\mathrm{H}-15$ & $\mathrm{H}-15$ \\
\hline
\end{tabular}




\begin{tabular}{|c|c|c|c|c|c|c|c|c|c|c|c|c|c|c|c|}
\hline 17 & $0.97 \mathrm{~s}$ & 22.2 & C- $12,13,14,15$ & & $\mathrm{H}-8,11 \mathrm{~b}$ & $0.98 \mathrm{~s}$ & 22.2 & C-12,14,15 & & $\mathrm{H}-8,14 \mathrm{a}, 15,16 \mathrm{a}$ & $0.98 \mathrm{~s}$ & 22.2 & C- $12,13,14,15$ & & H-12a,14a,15,16a, \\
\hline 18 & $1.04 \mathrm{~s}$ & 24.8 & $\mathrm{C}-3,4,5,19$ & & $\mathrm{H}-3 \mathrm{a}, 6$ & $1.04 \mathrm{~s}$ & 24.8 & $\mathrm{C}-3,4,5,19$ & $\mathrm{H}-2 \mathrm{a}, 3 \mathrm{a}$ & $\mathrm{H}-3 \mathrm{a}, 6,19 \mathrm{~b}$ & $1.03 \mathrm{~s}$ & 24.8 & $\mathrm{C}-3,4,5,19$ & $\mathrm{H}-2 \mathrm{a}, 3 \mathrm{a}$ & $\mathrm{H}-6,19 \mathrm{a}, 19 \mathrm{~b}$ \\
\hline 19a & $3.73 \mathrm{~d}(9.2)$ & 73.8 & C- $3,4,5,18,1^{\prime}$ & $\mathrm{H}-19 \mathrm{~b}$ & & $3.73 \mathrm{~d}(9.2)$ & 74.0 & & $H-19 b$ & $\mathrm{H}-10,2 \mathrm{~b}, 19 \mathrm{~b}, 1^{\prime}, 4^{\prime}$ & $3.77 \mathrm{~d}(9.2)$ & 73.6 & $\mathrm{C}-3,4,5,18,1^{\prime}$ & $\mathrm{H}-19 \mathrm{~b}$ & H-3a, $10,18,19 b, 1^{\prime}, 2^{\prime}$ \\
\hline $19 \mathrm{~b}$ & $3.04 \mathrm{~d}(9.2)$ & & C-3,4,5,18,1' & $\mathrm{H}-19 \mathrm{a}$ & & $3.07 \mathrm{~d}(9.2)$ & & & $\mathrm{H}-19 \mathrm{a}$ & $\mathrm{H}-10,18,19 \mathrm{a}, 1^{\prime}$ & $2.93 \mathrm{~d}(9.2)$ & & $\mathrm{C}-3,4,5,18,1^{\prime}$ & $\mathrm{H}-19 \mathrm{a}$ & $\mathrm{H}-8,18,19 \mathrm{a}, 1^{\prime}, 4^{\prime}$ \\
\hline 20 & $0.63 \mathrm{~s}$ & 12.1 & C-9,10 & & $\mathrm{H}-1 \mathrm{~b}, 11 \mathrm{a}$ & $0.63 \mathrm{~s}$ & 12.1 & C-8,9,10 & & $\mathrm{H}-7 \mathrm{~b}, 12 \mathrm{~b}$ & $0.63 \mathrm{~s}$ & 12.1 & C-9,10 & & $\mathrm{H}-7 \mathrm{~b}$, \\
\hline $1^{\prime}$ & $4.99 \mathrm{br} \mathrm{s}$ & 106.0 & C- $19,2^{\prime}, 3^{\prime}$ & & H-19a,19b,3' & $4.99 \mathrm{br} \mathrm{s}$ & 106.0 & C- $19,2^{\prime}, 3^{\prime}$ & & H-19a, 19b, $3^{\prime}$ & $4.93 \mathrm{br} \mathrm{s}$ & 107.6 & C- $19,2^{\prime}, 3^{\prime}$ & & H-19a,19b,3' \\
\hline $2^{\prime}$ & $4.81 \mathrm{~d}(2.4)$ & 85.8 & $\mathrm{C}-3^{\prime}, 2^{\prime}-\mathrm{CO}$ & & $\mathrm{H}-3 \mathrm{a}, 19 \mathrm{a}, 4^{\prime}$ & $4.82 \mathrm{dd}(0.8,2.8)$ & 84.5 & $\mathrm{C}-3^{\prime}, 2^{\prime}-\mathrm{CO}$ & $\mathrm{H}-3^{\prime}, 5^{\prime} \mathrm{a}$ & H-3a,19a, $4^{\prime}$ & $4.21 \mathrm{~d}(5.6)$ & 79.0 & $C-3^{\prime}$ & $\mathrm{H}-3^{\prime}, 5^{\prime} \mathrm{a}$ & H-3a, 19a, $4^{\prime}$ \\
\hline $3^{\prime}$ & $3.97 \mathrm{br} \mathrm{s}$ & 76.3 & C-4', $5^{\prime}$ & $\mathrm{H}-4^{\prime}$ & $\mathrm{H}-5^{\prime} \mathrm{a}, 5^{\prime} \mathrm{b}$ & $3.87 \mathrm{dd}(2.1,5.6)$ & 76.6 & C-4, $5^{\prime}$ & $\mathrm{H}-2^{\prime}, 4^{\prime}, 5^{\prime} \mathrm{a}$ & $\mathrm{H}-5^{\prime} \mathrm{a}, 5^{\prime} \mathrm{b}$ & $4.62 \mathrm{dd}(2.0,5.6)$ & 81.1 & $\mathrm{C}-4^{\prime}, 5^{\prime}, 3^{\prime}-\mathrm{CO}$ & $\mathrm{H}-2^{\prime}, 4^{\prime}, 5^{\prime} \mathrm{a}$ & $\mathrm{H}-5^{\prime} \mathrm{a}, 5^{\prime} \mathrm{b}$ \\
\hline $4^{\prime}$ & $4.07 \mathrm{~m}$ & 84.0 & $\mathrm{C}-2^{\prime}, 3^{\prime}$ & $\mathrm{H}-19 \mathrm{a}, 3^{\prime}, 5^{\prime} \mathrm{b}$ & $\mathrm{H}-19 \mathrm{~b}$ & $4.17 \mathrm{~d}(2.0)$ & 81.8 & $\mathrm{C}-2^{\prime}, 3^{\prime}$ & $\mathrm{H}-3^{\prime}$ & $\mathrm{H}-19 \mathrm{~b}, 2^{\prime}$ & $4.10 \mathrm{~d}(2.0)$ & 80.7 & $\mathrm{C}-2^{\prime}, 3^{\prime}$ & $\mathrm{H}-3^{\prime}$ & $\mathrm{H}-19 \mathrm{~b}, 2^{\prime}$ \\
\hline $5^{\prime} \mathrm{a}$ & $3.86 \mathrm{dd}(2.8,12.0)$ & 61.8 & $\mathrm{C}-3^{\prime}$ & $\mathrm{H}-4^{\prime}$ & $\mathrm{H}-3^{\prime}$ & $4.30 \mathrm{dd}(3.0,4.5)$ & 63.6 & $\mathrm{C}-3^{\prime}, 5^{\prime}-\mathrm{CO}$ & $\mathrm{H}-3^{\prime}, 4^{\prime}$ & $\mathrm{H}-3^{\prime}$ & $4.38 \mathrm{dd}(3.0,4.8)$ & 63.4 & $\mathrm{C}-3^{\prime}, 5^{\prime}-\mathrm{CO}$ & $\mathrm{H}-3^{\prime}, 4^{\prime}$ & $\mathrm{H}-3^{\prime}$ \\
\hline $5^{\prime} \mathrm{b}$ & $3.70 \mathrm{~d}(9.2)$ & & & & H-3a,19a,3' & $4.16 \mathrm{~d}(2.2)$ & & $5^{\prime}-\mathrm{CO}$ & $\mathrm{H}-3^{\prime}, 5^{\prime} \mathrm{a}$ & H-3a,19a,3' & $4.23 \mathrm{~d}(2.0)$ & & $5^{\prime}-\mathrm{CO}$ & $\mathrm{H}-3^{\prime}, 5^{\prime} \mathrm{a}$ & H-3a,19a,3' \\
\hline \multicolumn{16}{|l|}{ 2'-OAc } \\
\hline $\mathrm{COCH}_{3}$ & & 171.1 & & & & & 170.9 & & & & & & & & \\
\hline $\mathrm{COCH}_{3}$ & $2.08 \mathrm{~s}$ & 20.7 & $2^{\prime}-\mathrm{CO}$ & & & $2.08 \mathrm{~s}$ & 20.7 & $2^{\prime}-\mathrm{CO}$ & & & & & & & \\
\hline \multicolumn{16}{|l|}{$3^{\prime}-\mathrm{OAc}$} \\
\hline $\mathrm{COCH}_{3}$ & & & & & & & & & & & & 171.6 & & & \\
\hline $\mathrm{COCH}_{3}$ & & & & & & & & & & & $2.08 \mathrm{~s}$ & 20.6 & $3^{\prime}-\mathrm{CO}$ & & $\mathrm{H}-2^{\prime}, 4^{\prime}$ \\
\hline \multicolumn{16}{|l|}{$5^{\prime}-\mathrm{OAc}$} \\
\hline $\mathrm{COCH}_{3}$ & & & & & & & 170.6 & & & & & 170.5 & & & \\
\hline $\mathrm{COCH}_{3}$ & & & & & & $2.07 \mathrm{~s}$ & 20.7 & $5^{\prime}-\mathrm{CO}$ & & & $2.09 \mathrm{~s}$ & 20.7 & $5^{\prime}-\mathrm{CO}$ & & $\mathrm{H}-3^{\prime}, 4^{\prime}$ \\
\hline
\end{tabular}


Table S3. NMR spectral data of compounds 7 in $\mathrm{CDCl}_{3}\left[{ }^{1} \mathrm{H} 400 \mathrm{MHz},{ }^{13} \mathrm{C} 100 \mathrm{MHz}, \mathrm{TMS}, \quad \delta \quad(\mathrm{J}=\mathrm{Hz})\right]$

\begin{tabular}{|c|c|c|c|c|c|}
\hline \multirow[b]{2}{*}{ position } & \multirow[b]{2}{*}{${ }^{1} \mathrm{H}$} & \multicolumn{3}{|c|}{7} & \multirow[b]{2}{*}{ NOESY } \\
\hline & & ${ }^{13} \mathrm{C}$ & HMBC & ${ }^{1} \mathrm{H}-{ }^{1} \mathrm{H}$ COSY & \\
\hline $1 \mathrm{a}$ & $1.62 \mathrm{~m}$ & 23.0 & $\mathrm{C}-3$ & & $\mathrm{H}-2 \mathrm{~b}, 10$ \\
\hline $1 b$ & $1.56 \mathrm{~m}$ & & & & \\
\hline $2 \mathrm{a}$ & $1.62 \mathrm{~m}$ & 20.4 & $\mathrm{C}-3$ & & $\mathrm{H}-2 \mathrm{~b}, 3 \mathrm{a}$ \\
\hline $2 b$ & $1.35 \mathrm{~d}(10.4)$ & & $C-5,10$ & $\mathrm{H}-10$ & $\mathrm{H}-2 \mathrm{a}$ \\
\hline $3 a$ & $1.81 \mathrm{~d}(15.2)$ & 33.7 & & $\mathrm{H}-3 \mathrm{~b}$ & $\mathrm{H}-3 \mathrm{~b}, 18$ \\
\hline $3 b$ & $1.66 \mathrm{~d}(10.4)$ & & $\mathrm{C}-2$ & $\mathrm{H}-3 \mathrm{a}$ & H-3a, 18 \\
\hline 4 & & 51.8 & & & \\
\hline 5 & & 75.0 & & & \\
\hline $6 a$ & $2.09 \mathrm{dt}(4.4,13.2)$ & 33.6 & $\mathrm{C}-7,8$ & $\mathrm{H}-6 \mathrm{~b}, 7 \mathrm{a}$ & H-6b,7b \\
\hline $6 \mathrm{~b}$ & $1.72 \mathrm{~d}(14.0)$ & & C-5 & H-3a,6a & H-6a \\
\hline $7 \mathrm{a}$ & $1.46 \mathrm{~d}(20)$ & 25.0 & $\mathrm{C}-8$ & H-6a,7b & \\
\hline $7 b$ & $1.13 \mathrm{~d}(14.8)$ & & & H-6a,6b,7a & H-6a, $14 b$ \\
\hline 8 & $1.42 \mathrm{~m}$ & 40.7 & $\mathrm{C}-20$ & $\mathrm{H}-14 \mathrm{~b}$ & $\mathrm{H}-10,11 \mathrm{~b}$ \\
\hline 9 & & 36.3 & & & \\
\hline 10 & $1.77 \mathrm{~d}(14.0)$ & 49.3 & C-5,9,11,20 & $\mathrm{H}-2 \mathrm{~b}$ & $\mathrm{H}-8,11 \mathrm{~b}$ \\
\hline $11 \mathrm{a}$ & $1.53 \mathrm{~d}(8.0)$ & 34.8 & C-8 & $\mathrm{H}-11 \mathrm{~b}$ & $\mathrm{H}-20$ \\
\hline $11 b$ & $1.14 \mathrm{t}(13.8)$ & & $\mathrm{C}-20$ & H-7a, 11a & $\mathrm{H}-10$ \\
\hline $12 \mathrm{a}$ & $1.53 \mathrm{t}(15.2)$ & 31.9 & $\mathrm{C}-11,13,17$ & $\mathrm{H}-11 \mathrm{~b}$ & $\mathrm{H}-12 \mathrm{~b}, 15$ \\
\hline $12 b$ & $1.16 \mathrm{~d}(12.8)$ & & $\mathrm{C}-13,14,17$ & & $\mathrm{H}-12 \mathrm{a}$ \\
\hline 13 & & 36.6 & & & \\
\hline $14 \mathrm{a}$ & $1.38 \mathrm{~m}$ & 39.1 & & $\mathrm{H}-14 \mathrm{~b}$ & H-14b,15 \\
\hline $14 \mathrm{~b}$ & $0.98 \mathrm{~d}(11.6)$ & & $\mathrm{C}-17$ & $\mathrm{H}-14 \mathrm{a}$ & $\mathrm{H}-7 \mathrm{~b}, 14 \mathrm{a}$ \\
\hline 15 & $5.79 \mathrm{dd}(10.8,17.6)$ & 151.3 & C- $12,13,14,17$ & H-16a, 16b & H-12a, 16a, 16b, 17 \\
\hline $16 \mathrm{a}$ & $4.89 \mathrm{dd}(0.8,17.2)$ & 108.6 & C- 13,15 & H-15 & H-15,17 \\
\hline $16 \mathrm{~b}$ & $4.82 \mathrm{dd}(0.8,10.8)$ & & & & H-15 \\
\hline 17 & $1.02 \mathrm{~s}$ & 23.1 & C- $12,13,14,15$ & & H-11b,15.16a \\
\hline 18 & $1.20 \mathrm{~s}$ & 20.1 & C-4,5,6,19 & & $H-3 a, 3 b, 6 b$ \\
\hline 19 & & 182.3 & & & \\
\hline 20 & $0.88 \mathrm{~s}$ & 12.2 & C-8,10,11 & & $\mathrm{H}-11 \mathrm{a}$ \\
\hline
\end{tabular}


Table S4. NMR spectral data of compounds 8 in $\mathrm{CDCl}_{3}\left[{ }^{1} \mathrm{H} 400 \mathrm{MHz},{ }^{13} \mathrm{C} 100 \mathrm{MHz}, \mathrm{TMS}, \quad \delta(\mathrm{J}=\mathrm{Hz})\right]$

\begin{tabular}{|c|c|c|c|c|c|}
\hline \multirow[b]{2}{*}{ position } & \multicolumn{5}{|c|}{8} \\
\hline & ${ }^{1} \mathrm{H} N \overline{\mathrm{MR}}$ & ${ }^{13} \mathrm{C}$ NMR & HMBC & ${ }^{1} \mathrm{H}-{ }^{1} \mathrm{H} \operatorname{COSY}$ & $\overline{\text { NOESY }}$ \\
\hline $1 \mathrm{a}$ & $1.59 \mathrm{~d}(11.6)$ & 38.8 & $\mathrm{C}-5$ & $H-1 b, 2 b$ & $\mathrm{H}-1 \mathrm{~b}, 20$ \\
\hline $1 b$ & $0.83 \mathrm{~m}$ & & C-9 & $H-1 a, 2 b$ & H-1a,11a \\
\hline $2 \mathrm{a}$ & $1.60 \mathrm{~m}$ & 17.8 & & & \\
\hline $2 b$ & $1.47 \mathrm{~m}$ & & & & $\mathrm{H}-20$ \\
\hline $3 a$ & $1.36 \mathrm{~m}$ & 36.0 & C-4 & & \\
\hline $3 b$ & $1.27 \mathrm{~m}$ & & $\mathrm{C}-18$ & & \\
\hline 4 & & 36.9 & & & \\
\hline 5 & $1.23 \mathrm{~m}$ & 49.9 & C- $6,9,10$ & & $\mathrm{H}-1 \mathrm{~b}$ \\
\hline $6 a$ & $1.46 \mathrm{~m}$ & 19.8 & C-8,9 & $\mathrm{H}-1 \mathrm{a}$ & \\
\hline $6 b$ & $1.23 \mathrm{~m}$ & & $\mathrm{C}-10$ & & H-6a \\
\hline $7 \mathrm{a}$ & $1.72 \mathrm{~d}(10.4)$ & 42.7 & $\mathrm{C}-5,8,9$ & $\mathrm{H}-7 \mathrm{~b}$ & $\mathrm{H}-7 \mathrm{~b}, 17$ \\
\hline $7 b$ & $1.32 \mathrm{~m}$ & & $\mathrm{C}-5$ & $\mathrm{H}-7 \mathrm{a}$ & $\mathrm{H}-12 \mathrm{a}$ \\
\hline 8 & & 75.8 & & & \\
\hline 9 & $1.20 \mathrm{~m}$ & 58.4 & $\mathrm{C}-17$ & & $\mathrm{H}-7 \mathrm{~b}$ \\
\hline 10 & & 36.7 & & & \\
\hline $11 \mathrm{a}$ & $1.48 \mathrm{~m}$ & 15.8 & $\mathrm{C}-8$ & $\mathrm{H}-1 \mathrm{~b}$ & \\
\hline $11 \mathrm{~b}$ & $1.43 \mathrm{~m}$ & & & $\mathrm{H}-12 \mathrm{a}$ & \\
\hline $12 \mathrm{a}$ & $2.18 \mathrm{~m}$ & 34.8 & C-9, 11,13, & $\mathrm{H}-12 \mathrm{~b}$ & $H-12 b, 16$ \\
\hline $12 b$ & $1.44 \mathrm{~m}$ & & & $\mathrm{H}-12 \mathrm{a}$ & $\mathrm{H}-12 \mathrm{a}$ \\
\hline 13 & & 73.3 & & & \\
\hline 14 & $5.99 \mathrm{dd}(10.8,18.0)$ & 147.6 & $\mathrm{C}-12,16$ & $\mathrm{H}-15 \mathrm{a}, 15 \mathrm{~b}$ & H-15a, 15b,16,17 \\
\hline $15 \mathrm{a}$ & $4.95 \mathrm{~d}(18.0)$ & 109.5 & C- $13,14,16$ & $\mathrm{H}-14$ & $\mathrm{H}-14$ \\
\hline $15 b$ & $4.89 \mathrm{~d}(10.8)$ & & C- $13,14,16$ & $\mathrm{H}-14$ & $\mathrm{H}-14$ \\
\hline 16 & $1.11 \mathrm{~s}$ & 32.6 & C- $12,13,14,15$ & & $\mathrm{H}-12 \mathrm{a}$ \\
\hline 17 & $1.19 \mathrm{~s}$ & 23.8 & $\mathrm{C}-7,8,9$ & & H-7a, 14,20 \\
\hline 18 & $1.23 \mathrm{~s}$ & 29.6 & $\mathrm{C}-4$ & & \\
\hline $19 a$ & $3.27 \mathrm{~d}(9.2)$ & 76.8 & $\mathrm{C}-1^{\prime}$ & $\mathrm{H}-19 \mathrm{~b}$ & H-2b, $19 b, 20,1^{\prime}, 4^{\prime}$ \\
\hline $19 b$ & $3.13 \mathrm{~d}(9.2)$ & & $\mathrm{C}-1^{\prime}$ & $\mathrm{H}-19 \mathrm{a}$ & $\mathrm{H}-2 \mathrm{~b}, 19 \mathrm{a}, 20,1^{\prime}$ \\
\hline 20 & $0.73 \mathrm{~s}$ & 17.3 & C- $1,5,9,10$ & & $\mathrm{H}-1 \mathrm{a}, 2 \mathrm{~b}, 17$ \\
\hline $1^{\prime}$ & $4.99 \mathrm{br} \mathrm{s}$ & 105.4 & $\mathrm{C}-19,2^{\prime}, 3^{\prime}$ & & H-19a,19b,3' \\
\hline $2^{\prime}$ & $4.82 \mathrm{~d}(2.8)$ & 85.1 & $2^{\prime}-\mathrm{COCH}_{3}, \mathrm{C}-3^{\prime}$ & H-3' & $\mathrm{H}-4^{\prime}$ \\
\hline $3^{\prime}$ & $3.89 \mathrm{dd}(2.8,5.6)$ & 77.1 & $\mathrm{C}-2^{\prime}, 5^{\prime}$ & $\mathrm{H}-2^{\prime}, 4^{\prime}$ & $\mathrm{H}-1^{\prime}, 5^{\prime} \mathrm{a}$ \\
\hline $4^{\prime}$ & $4.18 \mathrm{~m}$ & 81.3 & & H-3' & H-19a, 2', 5'a \\
\hline $5^{\prime} \mathrm{a}$ & $4.30 \mathrm{~d}(7.2)$ & 63.5 & $\mathrm{C}-3^{\prime}, 4^{\prime}, 5^{\prime}-\mathrm{CO}$ & & $\mathrm{H}-3^{\prime}, 5^{\prime} \mathrm{b}$ \\
\hline $5^{\prime} \mathrm{b}$ & $4.16 \mathrm{~d}(7.2)$ & & $\mathrm{C}-3^{\prime}, 4^{\prime}, 5^{\prime}-\mathrm{CO}$ & & $\mathrm{H}-5^{\prime} \mathrm{a}$ \\
\hline \multicolumn{6}{|l|}{$2^{\prime}-\mathrm{OAc}$} \\
\hline $\mathrm{COCH}_{3}$ & & 171.2 & & & \\
\hline $\mathrm{COCH}_{3}$ & $2.11 \mathrm{~s}$ & 20.8 & $2^{\prime}-\mathrm{CO}$ & & \\
\hline \multicolumn{6}{|l|}{$5^{\prime}$-OAc } \\
\hline $\mathrm{COCH}_{3}$ & & 170.7 & & & \\
\hline $\mathrm{COCH}_{3}$ & $2.08 \mathrm{~s}$ & 20.8 & $5^{\prime}-\mathrm{CO}$ & & \\
\hline
\end{tabular}

